\title{
Permutation reconstruction from a few large patterns
}

\author{
Maria João Gouveia \\ Departamento de Matemática \\ Faculdade de Ciências \\ Universidade de Lisboa \\ Lisboa, Portugal \\ mjgouveia@fc.ul.pt
}

\author{
Erkko Lehtonen* \\ Centro de Matemática e Aplicações \\ Faculdade de Ciências e Tecnologia \\ Universidade Nova de Lisboa \\ Caparica, Portugal \\ e.lehtonen@fct.unl.pt
}

Submitted: Apr 29, 2021; Accepted: Aug 4, 2021; Published: Aug 27, 2021

(C) The authors. Released under the CC BY-ND license (International 4.0).

\begin{abstract}
Every permutation of rank $n \geqslant 5$ is reconstructible from any $\lceil n / 2\rceil+2$ of its $(n-1)$-patterns.
\end{abstract}

Mathematics Subject Classifications: 05A05

\section{Introduction}

The theory of permutation patterns and pattern avoidance has been an active field of research in the past decades. The fundamental notion of the theory is the pattern involvement relation. Writing a permutation $\pi \in S_{n}$ as a word $\pi_{1} \pi_{2} \ldots \pi_{n}$ where $\pi_{t}=\pi(t)$, a permutation $\tau \in S_{\ell}$ is called a pattern of $\pi$ if $\tau_{1} \tau_{2} \ldots \tau_{\ell}$ is order-isomorphic to some subword $\pi_{t_{1}} \pi_{t_{2}} \ldots \pi_{t_{\ell}}$ of $\pi$. For further information and background on permutation patterns, we refer the reader to Bóna [1] and Kitaev [4].

A reconstruction problem concerns whether a mathematical structure can be uniquely recovered from a collection of some derived structures that convey partial information on the original structure. The kind of reconstruction problems we discuss in this paper are exemplified by the famous unsolved problem in graph theory that concerns whether or not a finite simple graph is uniquely determined, up to isomorphism, by the collection of its one-vertex-deleted subgraphs. This problem was posed by Kelly [3] and Ulam [7], and it

*This work is funded by National Funds through the FCT - Fundação para a Ciência e a Tecnologia, I.P., under the scope of the project UIDB/00297/2020 (Center for Mathematics and Applications) and the project PTDC/MAT-PUR/31174/2017. 
is conjectured to hold for every graph with at least three vertices. Analogous reconstruction problems have been formulated and studied for many other kinds of mathematical structures.

The problem of reconstructing a permutation from its patterns has been considered by several authors. It is known that, for $n \geqslant 5$, every $n$-permutation is reconstructible from its multiset of $(n-1)$-patterns (Smith [6], Raykova [5]) and from its set of $(n-1)$ patterns (Ginsburg [2]). Raykova [5] and Smith [6] also considered decks (i.e. multisets) composed of $(n-k)$-patterns for $k \geqslant 1$ and, for every $k \geqslant 1$, they proved the existence of natural numbers $M$ such that all permutations of rank $n \geqslant M$ are reconstructible from their multisets of $(n-k)$-patterns. Although they did not determine $N_{k}$, the smallest of those numbers $M$, they could provide an upper bound and a lower bound for $N_{k}$.

When a permutation is reconstructible, its deck contains a sufficient amount of information for uniquely determining the permutation. Nonetheless, there may be some redundancy; perhaps some permutations can be reconstructed from only a few cards. Our main goal is to shed some light on how much information is needed for reconstruction, independently of the permutation we may consider. In this way we sharpen the previous results on the reconstructibility of permutations from patterns. In this paper we succeed to answer, for $k=1$, one of the open problems posed by Ginsburg in [2]: Can we find a non-trivial function $f_{k}:\left\{n \in \mathbb{N} \mid n \geqslant N_{k}\right\} \rightarrow \mathbb{N}$ so that $f_{k}(n)$ is the smallest integer $m$ such that every permutation $\pi \in S_{n}$ is uniquely determined by any of its partial $(n-k)$-decks of cardinality $m$ ? More precisely, here we prove that when $k=1$ the function $f_{k}$ is defined by $f_{1}(n)=\lceil n / 2\rceil+2$.

This paper is organized as follows. In Section 2, we introduce the basic notions and notation that will be needed throughout the paper. The main questions we are addressing in this paper are formulated precisely in Section 3. In Section 4, we look into certain special cases in which a permutation can be easily reconstructed from a few cards that contain the same monotone segment. Section 5 is devoted to our main result (Theorem 19): every permutation of rank $n \geqslant 5$ is reconstructible from any partial deck of cardinality $\lceil n / 2\rceil+2$. Its proof is constructive and yields a reconstruction algorithm. For the sake of illustrating the reconstruction method, several examples are given. We discuss some open problems in the final Section 6.

\section{Preliminaries}

Let the symbols $\mathbb{N}$ and $\mathbb{N}_{+}$denote the set of all nonnegative integers and the set of positive integers, respectively. For $n \in \mathbb{N}_{+}$, denote by $[n]$ the set $\{i \in \mathbb{N} \mid 1 \leqslant i \leqslant n\}$. For a set $A$ and $k \in \mathbb{N}$, we denote by $\left(\begin{array}{l}A \\ k\end{array}\right)$ the set of all $k$-element subsets of $A$.

A finite multiset $M$ over a nonempty set $X$ is a pair $\left(X, \chi_{M}\right)$, where $\chi_{M}: X \rightarrow \mathbb{N}$ is a map, called a multiplicity function, such that the support or underlying set $\operatorname{Supp}(M):=$ $\left\{x \in X \mid \chi_{M}(x) \neq 0\right\}$ is finite. We say that $x$ is an element of $M$ and we write $x \in M$ if $x \in \operatorname{Supp}(M)$. For $x \in X$, the number $\chi_{M}(x)$ is called the multiplicity of $x$ in $M$. In this paper only finite multisets are discussed and we will refer to them simply as multisets.

For a multiset $M$, the sum $\sum_{x \in X} \chi_{M}(x)$ is a well-defined natural number, and it is 
called the cardinality of $M$ and is denoted by $|M|$.

Let $M$ and $M^{\prime}$ be multisets over $X$. We say that $M$ is a submultiset of $M^{\prime}$ if $\chi_{M}(x) \leqslant$ $\chi_{M^{\prime}}(x)$ for all $x \in X$. The intersection of $M$ and $M^{\prime}$ is the multiset $M \cap M^{\prime}$ over $X$ given by the multiplicity function $\chi_{M \cap M^{\prime}}(x)=\min \left(\chi_{M}(x), \chi_{M^{\prime}}(x)\right)$ for all $x \in X$.

We may represent a multiset $M$ as a list enclosed in angle brackets where each element $x \in X$ occurs $\chi_{M}(x)$ times (the order of elements in the list does not matter), e.g., $\langle 0,0,1,1,1,1,2,3,4,4,4\rangle$. We also write $x^{m}$ to mean $m$ occurrences of $x$. (This will not create confusion because we will not be dealing with exponentiation in this paper.) Using this shorthand, the above multiset can be written briefly as $\left\langle 0^{2}, 1^{4}, 2,3,4^{3}\right\rangle$.

If $\left(a_{i}\right)_{i \in I}$ is a finite indexed family of elements of $X$, then we will write $\left\langle a_{i} \mid i \in I\right\rangle$ for the multiset in which the multiplicity of each element $x \in X$ equals $\left|\left\{i \in I \mid a_{i}=x\right\}\right|$.

For $n \in \mathbb{N}_{+}$, the set of all permutations of $[n]$ is denoted by $S_{n}$; these are called permutations of rank $n$. We may write a permutation $\pi \in S_{n}$ as a word $\pi_{1} \pi_{2} \ldots \pi_{n}$ where $\pi_{p}=\pi(p)$ for every $p \in[n]$.

For a nonempty subset $P=\left\{p_{1}, p_{2}, \ldots, p_{\ell}\right\} \subseteq[n]$ with $p_{1}<p_{2}<\cdots<p_{\ell}$, we denote by $\pi_{P}$ the permutation $\tau \in S_{\ell}$ such that the words $\pi_{p_{1}} \pi_{p_{2}} \ldots \pi_{p_{\ell}}$ and $\tau_{1} \tau_{2} \ldots \tau_{\ell}$ are orderisomorphic (with respect to the natural order of integers); when $P=[n] \backslash\{p\}$ we write $\pi-p$ instead. If $\tau$ is such a permutation $\pi_{P}$, then $\tau$ is called a pattern of $\pi$ and $\pi$ is said to involve $\tau$. We write $\tau \leqslant \pi$ when $\tau$ is a pattern of $\pi$.

Let $\pi \in S_{n}$. The reverse and the complement of $\pi$ are the permutations $\pi^{\mathrm{r}}$ and $\pi^{\mathrm{c}}$ in $S_{n}$, respectively, given by the rules $\pi^{\mathrm{r}}(t)=\pi(n-t+1)$ and $\pi^{\mathrm{c}}(t)=n-\pi(t)+1$, for all $t \in[n]$. It is well known that the pattern involvement relation is preserved under reverses and complements of permutations, as well as under taking inverses (i.e., if $\tau \leqslant \pi$, then $\tau^{\mathrm{r}} \leqslant \pi^{\mathrm{r}}, \tau^{\mathrm{c}} \leqslant \pi^{\mathrm{c}}$, and $\left.\tau^{-1} \leqslant \pi^{-1}\right)$.

For a permutation $\pi \in S_{n}$ and $k \in \mathbb{N}_{+}$, the $(n-k)$-deck of $\pi$ is the multiset $\operatorname{deck}_{n-k}(\pi):=\left\langle\pi_{P} \mid P \in\left(\begin{array}{c}{[n]} \\ n-k\end{array}\right)\right\rangle$. The elements of $\operatorname{deck}_{n-k}(\pi)$ are called the $(n-k)$-cards of $\pi$. If the number $n-k$ is clear from the context, we speak of the deck and the cards of $\pi$. Any submultiset of the deck of $\pi$ is called a partial deck of $\pi$.

A permutation $\pi \in S_{n}$ is reconstructible from its $(n-k)$-cards if for all $\sigma \in S_{n}$ it holds that $\operatorname{deck}_{n-k}(\pi)=\operatorname{deck}_{n-k}(\sigma)$ if and only if $\pi=\sigma$.

As we can easily observe from Lemma 6 , the ascending (identity) and the descending permutations of rank $n$, i.e., $\iota_{n}=12 \ldots n$ and $\delta_{n}=n(n-1) \ldots 1$, respectively, are the unique permutations that admit an $(n-1)$-deck with a unique element. Hence, for $n \geqslant 3$, $\iota_{n}$ and $\delta_{n}$ are examples of permutations that are reconstructible from their $(n-1)$-decks.

In Section 3 we present some constructions of permutations that preserve the existence of common cards. Some of those examples are particular cases of direct sums and skew sums of permutations. For $\sigma \in S_{m}$ and $\tau \in S_{n}$, the direct sum $\sigma \oplus \tau$ and the skew sum $\sigma \ominus \tau$ are the permutations of rank $m+n$ given by the rules

$$
\begin{aligned}
& (\sigma \oplus \tau)(t)= \begin{cases}\sigma(t), & \text { if } 1 \leqslant t \leqslant m, \\
\tau(t-m)+m, & \text { if } m+1 \leqslant t \leqslant m+n,\end{cases} \\
& (\sigma \ominus \tau)(t)= \begin{cases}\sigma(t)+m, & \text { if } 1 \leqslant t \leqslant m, \\
\tau(t-m), & \text { if } m+1 \leqslant t \leqslant m+n .\end{cases}
\end{aligned}
$$




\begin{tabular}{|c|c|c|c|c|c|}
\hline \multirow{2}{*}{\multicolumn{2}{|c|}{$\mathrm{C}_{n, k}$}} & \multicolumn{4}{|c|}{$k$} \\
\hline & & 1 & 2 & 3 & 4 \\
\hline \multirow{7}{*}{$n$} & 2 & $2^{*}$ & - & - & - \\
\hline & 3 & $3^{*}$ & $3^{*}$ & - & - \\
\hline & 1 & $4^{*}$ & $6^{*}$ & $4^{*}$ & - \\
\hline & 5 & $4^{\dagger}$ & $10^{*}$ & $10^{*}$ & $5^{*}$ \\
\hline & ; & 4 & $14^{\dagger}$ & $20^{*}$ & $15^{*}$ \\
\hline & 7 & 5 & 18 & $34^{\dagger}$ & $35^{*}$ \\
\hline & 8 & 5 & $?$ & $?$ & $?$ \\
\hline
\end{tabular}

Table 1: Values of $\mathrm{C}_{n, k}$ for small parameters $n$ and $k$. Asterisks indicate values equal to $\left(\begin{array}{c}n \\ n-k\end{array}\right)$, and daggers indicate values equal to $\left(\begin{array}{c}n \\ n-k\end{array}\right)-1$.

\section{Common partial decks}

We are now ready to formulate the main question that we are addressing in this paper.

Definition 1. For $n, k \in \mathbb{N}_{+}$, with $n>k$, define $H_{k}(n)$ to be the smallest integer $m$ such that every permutation $\pi \in S_{n}$ is uniquely determined by any of its partial $(n-k)$-decks of cardinality $m$.

Does the number $H_{k}(n)$ exist for given $n, k \in \mathbb{N}_{+}$? It is clear that $H_{k}(n)$ exists if and only if every permutation of rank $n$ is reconstructible from its $(n-k)$-deck, and that $H_{k}(n) \leqslant\left(\begin{array}{c}n \\ n-k\end{array}\right)$ in this case. As explained in the introduction, for every $k \in \mathbb{N}_{+}$, there exists a smallest number $N_{k}$ such that every permutation of rank $n \geqslant N_{k}$ is reconstructible from its $(n-k)$-deck (see Smith [6] and Raykova [5]). Consequently, $H_{k}(n)$ exists for every $n \geqslant N_{k}$, given $k \in \mathbb{N}_{+}$. In particular, $H_{1}(n)$ exists for $n \geqslant 5$ and $H_{2}(n)$ exists for $n \geqslant 6$ since $N_{1}=5$ (see Smith [6]) and $N_{2}=6$ (see Raykova [5]).

Problem 2. Given $n, k \in \mathbb{N}_{+}$such that $n \geqslant N_{k}$, what is the value of $H_{k}(n)$ ?

Definition 3. For $n, k \in \mathbb{N}$ with $1 \leqslant k<n$, define $\mathrm{C}_{n, k}$ to be the largest number $m$ for which there exist $\pi, \sigma \in S_{n}$ such that $\pi \neq \sigma$ but $\pi$ and $\sigma$ have $m$ common $(n-k)$-cards, i.e., $\left|\operatorname{deck}_{n-k}(\pi) \cap \operatorname{deck}_{n-k}(\sigma)\right|=m$.

Clearly, every permutation of rank $n$ is reconstructible from its $(n-k)$-deck if and only if $\mathrm{C}_{n, k}<\left(\begin{array}{c}n \\ n-k\end{array}\right)$. It follows immediately from the definitions that $H_{k}(n)=\mathrm{C}_{n, k}+1$ whenever $n \geqslant N_{k}$.

In Table 1 we present the numbers $C_{n, k}$ for small values of $n$ and $k$ that were discovered by an exhaustive computer search. Values equal to $\left(\begin{array}{c}n \\ n-k\end{array}\right)$ are indicated with asterisks - this means that not every permutation of rank $n$ is reconstructible from its $(n-k)$-deck. Values equal to $\left(\begin{array}{c}n \\ n-k\end{array}\right)-1$ are indicated with daggers - this means that there exist permutations of rank $n$ such that all $(n-k)$-cards are necessary for unique reconstructibility. 
Focusing on the case $k=1$, the pairs of distinct $n$-permutations with the maximum number $C_{n, 1}$ of common $(n-1)$-cards were determined for $2 \leqslant n \leqslant 8$; all such pairs are presented in Table 2, one pair in each row. Note that for $n \leqslant 4$, the listed permutations are precisely the permutations that are not reconstructible from $(n-1)$-decks.

At this point, we would like to draw the reader's attention to the following facts. On the one hand, the computational evidence seems to suggest that $C_{n, 1}=\lceil n / 2\rceil+1$ and hence $H_{1}(n)=\lceil n / 2\rceil+2$ for all $n \geqslant 5$. On the other hand, by noticing that

- for $n=2 k$, the two distinct permutations $\left(\iota_{k-1} \ominus 1\right) \oplus \iota_{k}$ and $\left(\iota_{k} \ominus 1\right) \oplus \iota_{k-1}$ have $\lceil n / 2\rceil+1$ common $(n-1)$-cards, and

- for $n=2 k+1$, the two distinct permutations $\iota_{k-1} \oplus \delta_{2} \oplus \iota_{k}$ and $\iota_{k} \oplus \delta_{2} \oplus \iota_{k-1}$ have $\lceil n / 2\rceil+1$ common $(n-1)$-cards,

we may immediately conclude that $C_{n, 1} \geqslant\lceil n / 2\rceil+1$; consequently, $H_{1}(n) \geqslant\lceil n / 2\rceil+2$ for all $n \geqslant 5$. In the next section, we are going to show that this lower bound is actually exact.

We end this section by highlighting some constructions of permutations that preserve the existence of common cards. For $\sigma, \tau \in S_{n}$, the following holds:

1. For every $p \in[n], \sigma^{\mathrm{r}}-p=(\sigma-(n-p+1))^{\mathrm{r}}$ and $\sigma^{\mathrm{c}}-p=(\sigma-p)^{\mathrm{c}}$.

2. For every $i \in[n], \sigma^{-1}-i=\left(\sigma-\sigma^{-1}(i)\right)^{-1}$.

3. If $\sigma$ and $\tau$ have $m$ common $(n-1)$-cards, then each of the pairs $\left(\sigma^{\mathrm{r}}, \tau^{\mathrm{r}}\right),\left(\sigma^{\mathrm{c}}, \tau^{\mathrm{c}}\right)$, and $\left(\sigma^{-1}, \tau^{-1}\right)$ also has $m$ common $(n-1)$-cards.

4. If $\sigma$ and $\tau$ have $m$ common $(n-1)$-cards, then the following pairs of $(n+1)$ permutations have $m$ common $n$-cards: $(1 \oplus \sigma, 1 \oplus \tau),(1 \ominus \sigma, 1 \ominus \tau),(\sigma \oplus 1, \tau \oplus 1)$, and $(\sigma \ominus 1, \tau \ominus 1)$.

It is not difficult to verify that the pairs of permutations of Table 2 for $n \geqslant 5$ can be built from permutations of lower rank by using some of these constructions. (Note that for $n=5$ we make use of the non-reconstructible pairs of 4 -permutations.)

\section{Monotone segments and their relevance for reconstructibility}

In this section we highlight some cases where the presence of a monotone segment in cards of a permutation is relevant to, and sometimes enough for, its reconstruction.

Given words $v$ and $w$ over some alphabet $A$, we write $v \sqsubseteq w$ if $v$ is a consecutive subword of $w$, i.e., there exist (possibly empty) words $x$ and $y$ over $A$ such that $x v y=w$. Recall that we may write a permutation $\pi \in S_{n}$ as a word $\pi_{1} \pi_{2} \ldots \pi_{n}$ over $[n]$. We call a nonempty consecutive subword of $\pi_{1} \pi_{2} \ldots \pi_{n}$ a segment of $\pi$. We denote the segment $\pi_{s} \pi_{s+1} \ldots \pi_{t}$ by $\pi[s, t]$. If $\tau$ and $\tau^{\prime}$ are segments of $\pi$ and $\tau \sqsubseteq \tau^{\prime}$, then we say that $\tau$ is a subsegment of $\tau^{\prime}$ and $\tau$ is a proper subsegment of $\tau^{\prime}$ if $\tau \sqsubseteq \tau^{\prime}$ and $\tau \neq \tau^{\prime}$. A segment $\pi[s, t]$ is ascending if $\pi_{i+1}=\pi_{i}+1$ for all $i \in[s, t-1]$, and $\pi[s, t]$ is descending if $\pi_{i+1}=\pi_{i}-1$ 


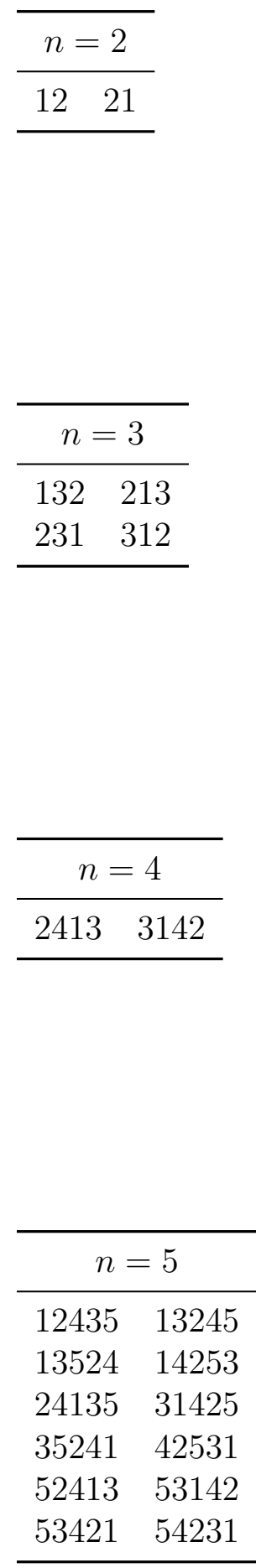

\begin{tabular}{ll}
\hline \multicolumn{2}{c}{$n=6$} \\
\hline 123546 & 124356 \\
123564 & 124563 \\
123645 & 126345 \\
124356 & 132456 \\
124356 & 132546 \\
124635 & 125364 \\
135246 & 142536 \\
146352 & 153642 \\
163524 & 164253 \\
164532 & 165342 \\
214635 & 215364 \\
231456 & 234156 \\
235461 & 243561 \\
241356 & 314256 \\
241365 & 314265 \\
312456 & 412356 \\
352416 & 425316 \\
352461 & 425361 \\
365421 & 465321 \\
463512 & 536412 \\
463521 & 536421 \\
524136 & 531426 \\
534216 & 542316 \\
543621 & 546321 \\
562413 & 563142 \\
612435 & 613245 \\
613524 & 614253 \\
624135 & 631425 \\
635241 & 642531 \\
645231 & 653421 \\
645321 & 653421 \\
651432 & 654132 \\
652413 & 653142 \\
653214 & 654213 \\
653421 & 654231 \\
\hline &
\end{tabular}

\begin{tabular}{ll}
\hline \multicolumn{2}{c}{$n=7$} \\
\hline 1235467 & 1243567 \\
7645321 & 7653421 \\
\hline
\end{tabular}

\begin{tabular}{ll}
\hline \multicolumn{2}{c}{$n=8$} \\
\hline 12346578 & 12354678 \\
12346785 & 12356784 \\
12348567 & 12384567 \\
12354678 & 12435678 \\
18756432 & 18764532 \\
23415678 & 23451678 \\
23465781 & 23546781 \\
41235678 & 51234678 \\
48765321 & 58764321 \\
76453218 & 76534218 \\
76548321 & 76584321 \\
81235467 & 81243567 \\
87564321 & 87645321 \\
87615432 & 87651432 \\
87643215 & 87653214 \\
87645321 & 87653421 \\
\hline
\end{tabular}

Table 2: Permutations of rank $n$ with $C_{n, 1}$ common $(n-1)$-cards, for $2 \leqslant n \leqslant 8$. 
for all $i \in[s, t-1]$. A segment is monotone if it is either ascending or descending. An ascending (descending) segment is maximal if it is not a proper subsegment of any ascending (descending) segment. The length of a segment $\pi[s, t]$ is defined as $t-s+1$, that is, the cardinality of the interval $[s, t]$. A segment is nontrivial if its length is greater than 1 . The initial and the final values of a segment $\pi[s, t]$ are $\pi_{s}$ and $\pi_{t}$, respectively. We sometimes view a segment $\pi[s, t]$ as the set $\left\{\pi_{i} \mid i \in[s, t]\right\}$ of its elements, and with slight misuse of notation, the meaning of expressions such as $i \in \pi[s, t]$ or $I \subseteq \pi[s, t]$ will be clear.

Henceforth we will consider mainly $(n-1)$-cards and (partial) $(n-1)$-decks of an $n$-permutation; therefore we will refer to them simply as cards and (partial) decks.

The following notation will be used to specify $(n-1)$-cards of an $n$-permutation. For $\pi \in S_{n}$ and $p \in[n]$, we denote by

- $\pi \downarrow i$ the $(n-1)$-permutation obtained by deleting from $\pi$ the entry with value $i$, i.e., if $p=\pi^{-1}(i)$, then $\pi \downarrow i=\pi-p$

and we recall that

- $\pi-p$ denotes the $(n-1)$-permutation obtained by deleting from $\pi$ the entry at the $p$-th position, i.e., the $(n-1)$-permutation order-isomorphic to $\pi_{1} \ldots \pi_{p-1} \pi_{p+1} \ldots \pi_{n}$.

In other words, $\pi \downarrow i=\pi_{[n] \backslash\left\{\pi^{-1}(i)\right\}}$ and $\pi-p=\pi_{[n] \backslash\{p\}}$. More generally, for a subset $J \subseteq[n]$, we define $\pi-J:=\pi_{[n] \backslash J}$ and $\pi \downarrow J:=\pi_{[n] \backslash \pi^{-1}(J)}$. For a permutation $\pi \in S_{n}$ and a set $I \subseteq[n]$, we denote by $\operatorname{deck}_{I}(\pi)$ the partial deck $\langle\pi \downarrow i \mid i \in I\rangle$.

Any permutation $\pi$ can be recovered when $\pi^{-1}(i)$ and $\pi \downarrow i$ are known for some $i \in[n]$, as is made explicit in the next lemma. For any $\tau \in S_{n-1}$ and $p, v \in[n]$, we denote by $\tau \uparrow_{p} v$ the permutation in $S_{n}$ obtained by adding 1 to all entries greater than or equal to $v$ and inserting at position $p$ an entry with value $v$, more precisely, $\tau \uparrow_{p} v=a_{1} \ldots a_{n}$, where

$$
a_{t}= \begin{cases}\tau(t), & \text { if } t<p \text { and } \tau(t)<v, \\ \tau(t)+1, & \text { if } t<p \text { and } \tau(t) \geqslant v, \\ v, & \text { if } t=p, \\ \tau(t-1), & \text { if } t>p \text { and } \tau(t)<v, \\ \tau(t-1)+1, & \text { if } t>p \text { and } \tau(t) \geqslant v .\end{cases}
$$

More generally, if $\tau=j_{1} j_{2} \ldots j_{m} \in S_{m}, p \in[m+1]$, and $\sigma=k_{1} k_{2} \ldots k_{\ell}$ is a word over $[n]$ with $n:=m+\ell$, then we define $\tau \uparrow_{p} \sigma$ as the permutation $a_{1} a_{2} \ldots a_{n} \in S_{n}$, where $a_{p} a_{p+1} \ldots a_{p+\ell-1}=\sigma$ and $a_{1} \ldots a_{p-1} a_{p+\ell} \ldots a_{n}$ is order-isomorphic to $j_{1} j_{2} \ldots j_{m}$.

Lemma 4. For any $\pi \in S_{n}$ and $i \in[n]$, we have $(\pi \downarrow i) \uparrow_{\pi^{-1}(i)} i=\pi$.

Proof. This is clear from the definitions.

Let us recall here a useful fact about iterated deletions of entries from a permutation.

Lemma 5 (Ginsburg [2, Lemma 1(vi)]). Let $\pi \in S_{n}$ and $p, q \in[n]$ with $p<q$. Then $(\pi \downarrow q) \downarrow p=(\pi \downarrow p) \downarrow(q-1)$. 
The existence of nontrivial monotone segments in a permutation is directly connected with the existence of cards of multiplicity greater than 1 in its deck.

Lemma 6 (Ginsburg [2, Lemma 1(iv)]). Let $\pi \in S_{n}$ and $s, t \in[n]$ with $s \leqslant t$. Then $\pi-s=\pi-t$ if and only if $\pi[s, t]$ is a monotone segment in $\pi$.

Lemma 7. Let $\pi \in S_{n}, I \subseteq[n]$, and $D:=\operatorname{deck}_{I}(\pi)$.

(i) The partial deck $D$ comprises a single card $\tau$ with multiplicity $|I|$ if and only if there is a monotone segment $\pi[s, t]$ of $\pi$ such that $I \subseteq \pi[s, t]$.

(ii) Assume that the condition of statement (i) holds and $|I| \geqslant 2$. If $\pi[s, t]$ is an ascending segment in $\pi$, then $\pi[s, t-1]=\tau[s, t-1]$ is an ascending segment in $\tau$ and $\pi=\tau \uparrow_{s} \tau(s)$. If $\pi[s, t]$ is a descending segment in $\pi$, then $\pi[s, t-1]=\tau[s, t-1]$ is a descending segment in $\tau$ and $\pi=\tau \uparrow_{t} \tau(t-1)$.

(iii) Assume that the condition of statement (i) holds and $|I| \geqslant\lceil n / 2\rceil+1$. Then $\tau$ has a unique maximal monotone segment $\pi[u, v]$ of length at least $\lceil n / 2\rceil$, and $I \subseteq$ $\pi[u, v+1]$.

Proof. (i) This follows immediately from Lemma 6.

(ii) This follows from a straightforward verification.

(iii) By (i), $\pi$ has a monotone segment $\pi[s, t]$ of length at least $\lceil n / 2\rceil+1$ such that $I \subseteq \pi[s, t]$; hence $\tau[s, t-1]$ is a monotone segment in $\tau$ and has length at least $\lceil n / 2\rceil$. If $\tau$ had two distinct maximal monotone segments of length at least $\lceil n / 2\rceil$, then $\tau$ would have rank at least $2\lceil n / 2\rceil \geqslant n$, a contradiction.

The above Lemma 7 asserts that any card $\tau$ of multiplicity $m \geqslant 2$ in a partial deck of $\pi$ arises by removing entries from a monotone segment of length $\ell \geqslant m+1$, and $\tau$ has consequently a monotone segment of length $\ell-1 \geqslant m$. The following lemma is a kind of converse statement and allows immediate reconstruction of $\pi$ in the case when the partial deck contains a card with large multiplicity and a unique long monotone segment.

For a segment $\sigma=k_{1} k_{2} \ldots k_{q}$, denote by $\sigma^{*}$ the word obtained from $\sigma$ by deleting its largest entry, and let $\sigma^{-}:=\left(k_{1}-1\right)\left(k_{2}-1\right) \ldots\left(k_{q}-1\right)$.

Proposition 8. Let $\pi \in S_{n}$, and let $D$ be a partial deck of $\pi$. Assume that $D$ contains a card $\tau$ of multiplicity $m \geqslant 3$ such that $\tau$ has a unique maximal monotone segment $\tau[u, v]=\sigma=k_{1} \ldots k_{q}$ of length at least $m-1$. If $\sigma$ is ascending, then $\pi=\tau \uparrow_{u} \tau(u)$. If $\sigma$ is descending, then $\pi=\tau \uparrow_{u}(\tau(u)+1)$.

Proof. Since $\tau$ has multiplicity $m$, it follows from Lemma 7 (i) that $\pi$ must contain a monotone segment $\lambda$ of length at least $m$ such that $\tau=\pi \downarrow i$ for any $i \in \lambda$.

Consequently, $\tau[u, v-1]=\lambda^{*}$ is a monotone segment in $\tau$, and it has length at least $m-1$. Since $\tau$ has a unique maximal monotone segment of length at least $m-1$, it holds that $\sigma=\lambda^{*}$. We have $\tau=\pi \downarrow \pi(u)$. By Lemma 4, if $\sigma$ (equivalently, $\lambda$ ) is ascending, then $\pi=(\pi \downarrow \pi(u)) \uparrow_{u} \pi(u)=\tau \uparrow_{u} k_{1}=\tau \uparrow_{u} \tau(u)$. If $\sigma$ (equivalently, $\lambda$ ) is descending, then $\pi=(\pi \downarrow \pi(u)) \uparrow_{u} \pi(u)=\tau \uparrow_{u}\left(k_{1}+1\right)=\tau \uparrow_{u}(\tau(u)+1)$. 
We will now develop a result (Proposition 12) which, together with its dual, obtained by reversing permutations, allows us to reduce the reconstruction of a permutation $\pi$ of rank $n$ to the reconstruction of a permutation of rank $n-q \leqslant n-2$ whenever the cards of a partial deck of $\pi$ contain the same maximal increasing or decreasing sequence of length $q$.

Definition 9. Let $\pi \in S_{n}$, and assume that $\pi[u, v]=k_{1} k_{2} \ldots k_{q}=: \sigma$ and $\pi\left[u^{\prime}, v^{\prime}\right]=$ $k_{1}^{\prime} k_{2}^{\prime} \ldots k_{r}^{\prime}=: \sigma^{\prime}$ are maximal ascending segments in $\pi$. We say that $i \in[n]$ is critical for $\left(\sigma, \sigma^{\prime}\right)$ in $\pi$, if one of the following conditions holds:

(1) $u^{\prime}=v+1, i=k_{q}+1, k_{1}^{\prime}=k_{q}+2$,

(2) $u^{\prime}=v+2, k_{1}^{\prime}=k_{q}+1, i=\pi(v+1)$.

In this case, we also say that $i$ is (upper) critical for $\sigma$ and (lower) critical for $\sigma^{\prime}$.

Remark 10. By definition, a maximal ascending segment $\pi[u, v]=k_{1} k_{2} \ldots k_{q}$ may have at most two upper critical points, and there are exactly two upper critical points if and only if $\pi(v+1)=k_{q}+2$ and $\pi(v+2)=k_{q}+1$. In this case, $k_{q}+2$ is critical for $\left(\pi[u, v], k_{q}+1\right)$ and $k_{q}+1$ is critical for $\left(\pi[u, v], k_{q}+2\right)$; moreover, $\left(k_{q}+2\right)\left(k_{q}+1\right)$ is a maximal descending segment in $\pi$, and hence $\pi \downarrow\left(k_{q}+1\right)=\pi \downarrow\left(k_{q}+2\right)$. Similarly, $\pi[u, v]$ may have at most two lower critical points, and there are exactly two lower critical points if and only if $\pi(u-1)=k_{1}-2$ and $\pi(u-2)=k_{1}-1$. In this case, $k_{1}-2$ is critical for $\left(\pi[u, v], k_{1}-1\right)$ and $k_{1}-1$ is critical for $\left(\pi[u, v], k_{1}-2\right)$; moreover, $\left(k_{1}-1\right)\left(k_{1}-2\right)$ is a maximal descending segment in $\pi$, and hence $\pi \downarrow\left(k_{1}-1\right)=\pi \downarrow\left(k_{1}-2\right)$.

Observe that if $i$ is critical for $\left(\mu, \mu^{\prime}\right)$ in $\pi$, then in the card $\pi \downarrow i$, the ascending segments $\mu$ and $\mu^{\prime}$ are merged and are part of a longer ascending segment. This will be made more precise in the following lemma.

Lemma 11. Let $\pi \in S_{n}$, and assume that $\pi[u, v]=k_{1} k_{2} \ldots k_{q}=: \sigma$ is a maximal ascending segment in $\pi$.

(i) If $q \geqslant 2$, then for every $i \in \pi[u, v],(\pi \downarrow i)[u, v-1]=\sigma^{*}$ is a maximal ascending segment in $\pi \downarrow i$.

(ii) If $\pi^{-1}(i)<u$ and $i<k_{1}$, then $(\pi \downarrow i)[u-1, v-1]=\sigma^{-}$.

(iii) If $\pi^{-1}(i)<u$ and $i>k_{q}$, then $(\pi \downarrow i)[u-1, v-1]=\sigma$.

(iv) If $\pi^{-1}(i)>v$ and $i<k_{1}$, then $(\pi \downarrow i)[u, v]=\sigma^{-}$.

(v) If $\pi^{-1}(i)>v$ and $i>k_{q}$, then $(\pi \downarrow i)[u, v]=\sigma$.

(vi) The ascending segments in $\pi \downarrow i$ described in (ii)-(v) are maximal precisely unless $i$ is critical for $\sigma$ in $\pi$.

(vii) Consequently, $\sigma^{*}$ is an ascending segment in every card of $\pi$. 
(viii) If $\sigma^{\prime}=k_{1}^{\prime} k_{2}^{\prime} \ldots k_{r}^{\prime}$ is another maximal ascending segment in $\pi$ and $i$ is critical for $\left(\sigma, \sigma^{\prime}\right)$, then $\pi \downarrow i$ has an ascending segment $\lambda$ such that

- $\sigma \sigma^{\prime-} \sqsubseteq \lambda$ if $k_{1}^{\prime}=k_{q}+2$;

- $\sigma \sigma^{\prime} \sqsubseteq \lambda$ if $k_{1}^{\prime}=k_{q}+1$ and $i>k_{r}^{\prime}$;

- $\sigma^{-} \sigma^{-} \sqsubseteq \lambda$ if $k_{1}^{\prime}=k_{q}+1$ and $i<k_{1}$.

Proof. This follows from a straightforward verification.

Proposition 12. Assume $n \geqslant 5, D$ is a partial deck of a permutation $\pi \in S_{n}$, and $|D|=H(n)$. Assume that the cards in $D$ are not all equal, there is a card in $D$ that has a maximal ascending segment $\kappa=k_{1} k_{2} \ldots k_{q}$ with $2 \leqslant q \leqslant n-H(n)$, and in every card in $D$, either $\kappa$ or $\kappa^{-}$is a maximal ascending segment. Then the following statements hold.

(i) There is a maximal ascending segment $\lambda$ in $\pi$ such that the cards in $D$ are obtained by removing entries that do not lie in $\lambda$ and are not critical for $\lambda$ in $\pi$. Consequently, either $\lambda$ or $\lambda^{-}$is a maximal ascending segment in each card in $D$, and $\kappa \in\left\{\lambda, \lambda^{-}\right\}$.

(ii) Let $G:=\left\{g_{\tau} \mid \tau \in D\right\}$, where

$$
g_{\tau}:= \begin{cases}\left(k_{1}, \tau^{-1}\left(k_{1}\right)\right), & \text { if } \kappa \sqsubseteq \tau, \\ \left(k_{1}-1, \tau^{-1}\left(k_{1}-1\right)\right), & \text { if } \kappa^{-} \sqsubseteq \tau .\end{cases}
$$

Let $A:=\{a \mid \exists b(a, b) \in G\}, B:=\{b \mid \exists a(a, b) \in G\}, a^{*}:=\max A, b^{*}:=\max B$. Let

$$
\begin{aligned}
& j:= \begin{cases}a^{*}+1, & \text { if }|A|=1 \text { and } H(n) \leqslant a^{*}, \\
a^{*}, & \text { otherwise, }\end{cases} \\
& u:= \begin{cases}b^{*}+1, & \text { if }|B|=1 \text { and } H(n) \leqslant b^{*}, \\
b^{*}, & \text { otherwise, }\end{cases} \\
& v:=u+q-1,
\end{aligned}
$$

Then $\pi[u, v]$ is a maximal ascending segment in $\pi$ with initial value $j$ and length $q$.

(iii) Let $D^{\prime}:=\left\langle\tau^{\prime} \mid \tau \in D\right\rangle$, where

$$
\tau^{\prime}:= \begin{cases}\tau \downarrow \kappa, & \text { if } \kappa \sqsubseteq \tau, \\ \tau \downarrow \kappa^{-}, & \text {if } \kappa^{-} \sqsubseteq \tau .\end{cases}
$$

Then $D^{\prime}$ is a partial $(n-q-1)$-deck of $\pi \downarrow \pi[u, v] \in S_{n-q}$, where $u$ and $v$ are as in part (ii).

(iv) Let $u, v$, and $t$ be as in (ii) and $D^{\prime}$ be as in (iii). If $\theta:=\pi \downarrow \pi[u, v]$ is reconstructible from $D^{\prime}$, then $\pi$ is reconstructible from $D$ as $\theta \uparrow{ }_{u} \pi[u, v]$ 
Proof. (i) Let $\gamma$ be a card in $D$ that has a maximal ascending segment $\kappa$ satisfying the conditions of the proposition. The card $\gamma$ must arise in one of the following ways:

1. There is a maximal ascending segment $\lambda:=k_{1} k_{2} \ldots k_{q}\left(k_{q}+1\right)$ in $\pi$ such that $\gamma=$ $\pi \downarrow i$ for some (any) $i \in \lambda$.

2. There are maximal ascending segments $\mu$ and $\mu^{\prime}$ and a critical point $i$ for $\left(\mu, \mu^{\prime}\right)$ in $\pi$ such that $\gamma=\pi \downarrow i$ and $\mu \mu^{\prime-}, \mu \mu^{\prime}$, or $\mu^{-} \mu^{\prime-}$ is a subsegment of $\kappa$, according to Lemma 11(viii).

3. There is a maximal ascending segment $\lambda$ in $\pi$ such that $\kappa \in\left\{\lambda, \lambda^{-}\right\}$and $\gamma=\pi \downarrow i$ for some $i \in[n] \backslash \lambda$ that is not critical for $\lambda$ in $\pi$.

We claim that cases 1 and 2 are impossible.

Case 1 is not possible because of our assumption that the cards in $D$ are not all equal. Namely, by Lemma $11, \kappa=\lambda^{*}$ is an ascending segment in all cards, but it is not a maximal one in the cards distinct from $\gamma$, contradicting our hypothesis.

Suppose now, to the contrary, that case 2 occurs. Consider first the case when the length of $\mu$ is at least 2. Since $\mu$ has at most 4 critical points in $\pi$ and $H(n) \geqslant 5$, there is a card $\delta \in D$ such that $\delta=\pi \downarrow j$ for some $j$ that is not critical for $\mu$ in $\pi$. By Lemma 11, $\mu, \mu^{-}$, or $\mu^{*}$ is a maximal ascending segment in $\delta$. Since each one of $\mu, \mu^{-}$and $\mu^{*}$ has a nonempty intersection with both $\kappa$ and $\kappa^{-}$and is also shorter than them, neither $\kappa$ nor $\kappa^{-}$is an ascending segment in $\delta$, contradicting our assumptions. The case when the length of $\mu^{\prime}$ is at least 2 is treated similarly.

Consider now the case when both $\mu$ and $\mu^{\prime}$ have length 1 , say $\mu=a$ and $\mu^{\prime}=b$ (and hence $b \in\{a+1, a+2\})$; then $a(a+1)$ or $(a-1) a$ is a subsegment of $\kappa$. Observe first that if $D$ contained a card of the form $\pi \downarrow p$ for some $p$ that is neither a nor a critical point of $\mu$, then $\mu=a$ or $\mu^{-}=a-1$ would be a maximal ascending segment in $\pi \downarrow p$ by Lemma 11, and therefore neither $\kappa$ nor $\kappa^{-}$would be a maximal ascending segment in $\pi \downarrow p$, which would contradict the hypotheses of the proposition. Since $\mu$ has at most 4 critical points and $H(n) \geqslant 5$, we are left with the situation where $H(n)=5, \mu$ has exactly 4 critical points, and $\pi \downarrow a \in D$. Then necessarily $(a-1)(a-2) a(a+2)(a+1) \sqsubseteq \pi$; hence $(a-1)(a-2)(a+1) a \sqsubseteq \pi \downarrow a$, so neither $\kappa$ nor $\kappa^{-}$is a maximal ascending segment in $\pi \downarrow a$. We have reached a contradiction also in this case.

We conclude that case 3 is the only one possible. Furthermore, we see easily with the help of Lemma 11, by considering the length of the maximal ascending segment containing $\lambda^{*}$, that all cards in $D$ must be of the form $\pi \downarrow i$ for some $i$ that neither belongs to $\lambda$ nor is critical for $\lambda$. Therefore either $\lambda$ or $\lambda^{-}$is a maximal ascending segment in each card in $D$, and $\kappa \in\left\{\lambda, \lambda^{-}\right\}$.

(ii) Let $\lambda=\ell_{1} \ell_{2} \ldots \ell_{q}$ be the maximal ascending segment in $\pi$ provided by part (i), and assume that $\pi[u, v]=\lambda$. It is straightforward to verify that

- $\lambda^{-} \sqsubseteq \pi-i$ and $(\pi-i)^{-1}\left(\ell_{1}-1\right)=u-1$ if and only if $i<u$ and $\pi(i)<\ell_{1}$;

- $\lambda^{-} \sqsubseteq \pi-i$ and $(\pi-i)^{-1}\left(\ell_{1}-1\right)=u$ if and only if $i<u$ and $\pi(i)>\ell_{q}$; 
- $\lambda \sqsubseteq \pi-i$ and $(\pi-i)^{-1}\left(\ell_{1}\right)=u-1$ if and only if $i>v$ and $\pi(i)<\ell_{1}$;

- $\lambda \sqsubseteq \pi-i$ and $(\pi-i)^{-1}\left(\ell_{1}\right)=u$ if and only if $i>v$ and $\pi(i)>\ell_{q}$.

Therefore, we have $A \subseteq\left\{\ell_{1}, \ell_{1}-1\right\}$ and $B \subseteq\{u, u-1\}$. It is clear that if $|A|=2$, then $A=\left\{k_{1}, k_{1}-1\right\}=\left\{\ell_{1}, \ell_{1}-1\right\}$, so $j=a^{*}=k_{1}=\ell_{1}$. Assume now that $|A|=1$. Then all cards in $D$ are of the form $\pi-i$ with $\pi(i)<\ell_{1}$, or they are all of the form $\pi-i$ with $\pi(i)>\ell_{q}$. The former case holds if and only if $H(n)<\ell_{1}$, and the latter case holds if and only if $H(n)<n-\ell_{q}$ (both inequalities cannot hold simultaneously because otherwise we would have $n+4 \leqslant 2 H(n)<n-\left(\ell_{q}-\ell_{1}\right)<n$, a contradiction). In the former case, we have $A=\left\{\ell_{1}-1\right\}$ and $H(n) \leqslant \ell_{1}-1=a^{*}$, so $j=a^{*}+1=\ell_{1}$. In the latter case, we have $A=\left\{\ell_{1}\right\}$ and $H(n) \not \leq \ell_{1}-1$, so $j=a^{*}=\ell_{1}$. In a similar way we can show that $u=\pi^{-1}\left(\ell_{1}\right)$. Finally, since the length of $\kappa$ equals that of $\lambda$, we have for $v:=u+q-1$ that $\pi[u, v]=\lambda$ is a maximal ascending segment in $\pi$ that has initial walue $\ell_{1}=j$.

(iii) Since each $\tau \in D$ is of the form $\pi \downarrow j$ for some $j \in[n] \backslash\left[\ell_{1}, \ell_{q}\right]$, it follows from Lemma 5 that

- if $j<k_{1}$, then $(\pi \downarrow j) \downarrow \lambda^{-}=(\pi \downarrow \lambda) \downarrow j$,

- if $j>k_{q}$, then $(\pi \downarrow j) \downarrow \lambda=(\pi \downarrow \lambda) \downarrow(j-q)$.

Consequently, $D^{\prime}$ is an $(n-q-1)$-deck of $\pi \downarrow \lambda \in S_{n-q}$.

(iv) Since $H(n) \geqslant H(n-q), \theta:=\pi \downarrow \lambda$ is reconstructible from $D^{\prime}$. Then we obtain $\pi$ as $\theta \uparrow_{u}\left(\ell_{1} \ldots \ell_{q}\right)=(\pi \downarrow \lambda) \uparrow_{u} \lambda$.

\section{Reconstructing a permutation from a partial deck}

In this section, we show that for $n \geqslant 5$, every permutation of rank $n$ is uniquely determined by any of its partial $(n-1)$-decks of cardinality $H(n):=\lceil n / 2\rceil+2$. The proof is constructive and can be turned into a reconstruction algorithm. The idea is to aim at determining $\pi^{-1}(i)$ and $\pi \downarrow i$, for some $i \in[n]$, from the given partial deck of $\pi$, because these would enable us to recover $\pi$ by Lemma 4 . If this is not directly possible, we are nevertheless able to determine $\pi^{-1}(1)$ and to reconstruct $\pi \downarrow 1$ by a recursive application of the algorithm.

For a permutation $\pi \in S_{n}$, the pair $\left(\pi^{-1}(1), \pi^{-1}(2)\right)$ is referred to as the type of $\pi$. As described in the following lemma, the cards of $\pi$ have only a few possible types, and only three of them may occur as types of multiple cards. Furthermore, the relative order of 1 and 2 in $\pi$ and their adjacency can be determined from a few cards.

Lemma 13. Let $n \geqslant 5, \pi \in S_{n}, p:=\pi^{-1}(1)$ and $r:=\pi^{-1}(2)$. For each $t \in[n]$, let $p_{t}:=(\pi-t)^{-1}(1)$ and $r_{t}:=(\pi-t)^{-1}(2)$. 
(i) If $p<r$, then

$$
\left(p_{t}, r_{t}\right)= \begin{cases}(p-1, r-1) & \text { if } t<p, \\ (r-1, x) & \text { if } t=p, \\ (p, r-1) & \text { if } p<t<r, \\ (p, y) & \text { if } t=r, \\ (p, r) & \text { if } r<t,\end{cases}
$$

for some $x, y \in[n]$ that satisfy $x=y=\pi^{-1}(3) \leqslant p-1$ or $x=y=\pi^{-1}(3)-1 \geqslant r$ or $x=y-1$ with $y=\pi^{-1}(3) \in[p+1, r-1]$. If $r<p$, then

$$
\left(p_{t}, r_{t}\right)= \begin{cases}(p-1, r-1) & \text { if } t<r, \\ (p-1, x) & \text { if } t=r, \\ (p-1, r) & \text { if } r<t<p, \\ (r, y) & \text { if } t=p, \\ (p, r) & \text { if } p<t,\end{cases}
$$

for some $x, y \in[n]$ that satisfy $x=y=\pi^{-1}(3) \leqslant r-1$ or $x=y=\pi^{-1}(3)-1 \geqslant p$ or $x=y-1$ with $y=\pi^{-1}(3) \in[r+1, p-1]$.

(ii) We have $p<r$ if and only if $p_{t}<r_{t}$ for all $t \in[n] \backslash\{p, r\}$. Similarly, $r<p$ if and only if $r_{t}<p_{t}$ for all $t \in[n] \backslash\{p, r\}$.

(iii) We have $p=r-1$ if and only if $p_{t}=r_{t}-1$ for all but at most two indices $t \in[n]$. Moreover, $p \neq r-1$ if and only if $p_{t} \neq r_{t}-1$ for all but at most two indices $t \in[n]$.

Proof. (i) This follows from a straightforward verification.

(ii) This follows immediately from (i).

(iii) Necessity is clear by (i), because if $p=r-1$, then $p_{t}=r_{t}-1$ for all $t \in[n] \backslash\{p, r\}$ (note that the case $p<t<r$ does not occur here).

For sufficiency and for the last assertion we prove that $p \neq r-1$ implies that $p_{t} \neq r_{t}-1$ for all but at most two indices $t \in[n]$. Since $n \geqslant 5$, this means that the inequality holds for at least three indices $t \in[n]$.

So assume that $p \neq r-1$. We consider different cases. If $r<p$, then $r_{t}<p_{t}$ and hence $p_{t} \neq r_{t}-1$ for all $t \in[n] \backslash\{p, r\}$ by (ii). If $p<r-2$, then $p_{t}<r_{t}-1$ for all $t \in[n] \backslash\{p, r\}$ by (i). Consider finally the case $p=r-2$. Then $p_{t}<r_{t}-1$ for all $t \in[n] \backslash\{p, p+1, r\}$, and $p_{p+1}=r_{p+1}-1$. If $p_{r}=r_{r}-1$, then $\pi(p+1)=3$, and it follows that $\left(p_{p}, r_{p}\right)=(r-1, p)=(p+1, p)$, so $p_{p}>r_{p}$. If $p_{p}=r_{p}-1$, then $\pi(r+1)=3$, and it follows that $\left(p_{r}, r_{r}\right)=(p, r)=(p, p+2)$, so $p_{r}<r_{r}-1$. We conclude that in each case there are at most two indices $t \in[n]$ such that $p_{t}=r_{t}-1$.

Note that we have also proved that $p \neq r-1$ if and only if $p_{t} \neq r_{t}-1$ for all but at most two indices $t \in[n]$.

Remark 14. By Lemma 13(ii), we may decide whether $\pi^{-1}(1)<\pi^{-1}(2)$ or $\pi^{-1}(1)>\pi^{-1}(2)$ by comparing the relative order of 1 and 2 in the cards of $\pi$; they are in the same order 
as in $\pi$ with at most two exceptions. In fact, this condition can be checked by applying the majority rule to any five cards. Similarly, by Lemma 13(iii), we may decide whether 1 and 2 are adjacent in $\pi$, i.e., $\pi^{-1}(1)=\pi^{-1}(2)-1$, by checking whether 1 and 2 are adjacent in all cards of $\pi$ with at most two exception. Again, it is sufficient to check only five cards.

Note that $\pi^{-1}(1)$ is known as soon as we know $\left(\pi^{\mathrm{r}}\right)^{-1}(1)$. Since the pattern involvement relation is preserved and reflected under reversing permutations, we may consider $\pi^{\mathrm{r}}$ in place of $\pi$ and reverse all given cards when $\pi^{-1}(1)>\pi^{-1}(2)$. Therefore, from now on we assume that $\pi^{-1}(1)<\pi^{-1}(2)$.

Given a permutation $\tau$ with $\tau^{-1}(1)<\tau^{-1}(2)$, we denote by $\ell_{\tau}$ the length of the longest ascending segment of $\tau$ with initial value 1 . Note that $\ell_{\tau}=1$ if and only if 1 and 2 are not adjacent.

Let us first consider the case where 1 and 2 are adjacent in $\pi$.

Lemma 15. Let $n \geqslant 5, \pi \in S_{n}$ and $I \subseteq[n]$ with $|I|=H(n)$. For each $i \in[n]$, let $p_{i}:=(\pi \downarrow i)^{-1}(1), r_{i}:=(\pi \downarrow i)^{-1}(2)$ and $\ell_{i}:=\ell_{\pi \downarrow i}$. Let $L:=\left\langle\ell_{i} \mid i \in I\right\rangle$. Suppose that $\ell_{\pi}>1$ and let $\pi[p, t]$ be the longest ascending segment with initial value 1 in $\pi$ (i.e., $p=\pi^{-1}(1)$ and $\left.t=p+\ell_{\pi}-1\right)$.

(i) For $i \in[n]$, the following conditions are equivalent.

(a) $\pi \downarrow i=\pi \downarrow 1$.

(b) $i \in \pi[p, t]$.

(c) $\ell_{i}=\ell_{\pi}-1$.

(ii) For all $i \in[n] \backslash \pi[p, t]$, we have $\ell_{i} \geqslant \ell_{\pi}$, and $i \in\{\pi(t+1), \pi(t)+1\}$ whenever $\ell_{i}>\ell_{\pi}$. Moreover, if $i, j \in[n]$ are such that $i \neq j$ and $\ell_{i}, \ell_{j}>\ell_{\pi}$, then $\ell_{i}=\ell_{j}$, $\pi(t+1)=\pi(t)+2, \pi(t+2)=\pi(t)+1$ and $\pi \downarrow i=\pi \downarrow j$.

(iii) I has a nonempty intersection with both $\pi[1, p-1]$ and $\pi[p, n]$ if and only if there exist $i, j \in I$ such that $p_{i} \neq p_{j}$. In this case $p=\max \left\{p_{i}, p_{j}\right\}$ and $p-1=\min \left\{p_{i}, p_{j}\right\}$. Moreover, for any $i \in I$ with $p_{i}=p-1$, it holds that $\ell_{i}=\ell_{\pi}$.

(iv) $I \subseteq \pi[1, p-1]$ if and only if there is an $a \in[n-1]$ with $a \geqslant H(n)$ such that $p_{i}=a$ for all $i \in I$. In this case, $p=a+1$ and there is no $i \in I$ such that $\pi \downarrow i=\pi \downarrow 1$.

(v) $I \subseteq \pi[p, n]$ if and only if there is an $a \in[n-1]$ with $a \leqslant n-H(n)+1$ such that $p_{i}=a$ for all $i \in I$. In this case $p=a$.

Proof. Recall that $|I|=H(n)$ and note that $\ell_{\pi}>1$ implies that $\pi^{-1}(2)=p+1$. (i) The equivalence of (a) and (b) follows from Lemma 6, and the equivalence of (b) and (c) is clear.

(ii) By Lemma 11, we have $\ell_{i} \geqslant \ell_{\pi}$ whenever $i \in[n] \backslash \pi[p, t]$. Since $\pi(p)=1$, the strict inequality $\ell_{i}>\ell_{\pi}$ holds only if $i$ is an upper critical point for $\pi[p, t]$, which implies by Definition 9 that $i \in\{\pi(t+1), \pi(t)+1\}$. If $\pi[p, t]$ has two upper critical points, then, by 
Remark 10, $\pi(t+1)=\pi(t)+2, \pi(t+2)=\pi(t)+1$, and $\pi \downarrow \pi(t+1)=\pi \downarrow \pi(t+2)$, from which the claim follows immediately.

(iii) Recall that $p_{i}=(\pi \downarrow i)^{-1}(1)$ and $r_{i}=(\pi \downarrow i)^{-1}(2)$. Since $\pi^{-1}(2)=p+1$, by applying Lemma 13(i), we see that $p_{i} \in\{p-1, p\}$ for any $i \in I$. Moreover, for any $i, j \in I$, it holds that $p-1=p_{i}<p_{j}=p$ if and only if $\pi^{-1}(i)<p \leqslant \pi^{-1}(j)$, which is equivalent to $i \in \pi[1, p-1]$ and $j \in \pi[p, n]$. Finally, for any $i \in \pi[1, p-1]$, we have $\ell_{i}=\ell_{\pi}$ by part (ii).

(iv) and (v) By Lemma 13(i), the following holds:

- If $I \subseteq \pi[1, p-1]$, then $p-1 \geqslant H(n)$ and $p_{i}=p-1$ for all $i \in I$. This shows the necessity of the condition in (v) with $a=p-1$.

- If $I \subseteq \pi[p, n]$, then $n-p+1 \geqslant H(n)$ (and so $n-H(n)+1 \geqslant p$ ) and $p_{i}=p$ for all $i \in I$. This shows the necessity of the condition in (iv) with $a=p$.

Conversely, assume that there is an $a \in[n-1]$ such that $p_{i}=a$ for all $i \in I$. By part (iii), we have $I \subseteq \pi[1, p-1]$ (and so $a \geqslant H(n)$ ) or $I \subseteq \pi[p, n]$ (and so $a \leqslant n-H(n)+1$ ). Note that the conditions $a \geqslant H(n)$ and $a \leqslant n-H(n)+1$ cannot hold simultaneously, because if $H(n) \leqslant a \leqslant n-H(n)+1$, then $n \geqslant 2 H(n)-1=2(\lceil n / 2\rceil+2)-1 \geqslant n+3$, which is absurd. Therefore $a \geqslant H(n)$ implies $I \subseteq \pi[1, p-1]$ and $a \leqslant n-H(n)+1$ implies $I \subseteq \pi[p, n]$.

It follows immediately from (i) that when $a \geqslant H(n)$ there is no $i \in I$ such that $\pi \downarrow i=\pi \downarrow 1$.

Let us now consider the case when 1 and 2 are not adjacent in $\pi$.

Lemma 16. Let $n \geqslant 5, \pi \in S_{n}, I \subseteq[n]$ with $|I|=H(n)$. Let $p:=\pi^{-1}(1)$ and $r:=\pi^{-1}(2)$, and assume that $p<r-1$. For each $k \in[n]$, let $p_{k}:=(\pi \downarrow k)^{-1}(1)$ and $r_{k}:=(\pi \downarrow k)^{-1}(2)$.

(I) For all $k \in[n]$, it holds that $p_{k}=r-1$ if and only if $k=1$. In other words, $\pi \downarrow 1$ is the unique card of $\pi$ with 1 at position $r-1$.

(II) If $\alpha, \beta, \gamma, \delta \in[n]$ are distinct indices such that

$$
\left(p_{\alpha}, r_{\alpha}\right)=\left(p_{\beta}, r_{\beta}\right) \neq\left(p_{\gamma}, r_{\gamma}\right)=\left(p_{\delta}, r_{\delta}\right),
$$

then $p=\max \left\{p_{\alpha}, p_{\gamma}\right\}$ and $r-1=\min \left\{r_{\alpha}, r_{\gamma}\right\}$.

(III) Assume that there exists a unique pair $(a, b) \in[n-1] \times[n-1]$ such that there exist distinct $i, j \in I$ with $\left(p_{i}, r_{i}\right)=\left(p_{j}, r_{j}\right)=(a, b)$. Let $E:=\left\{\left(p_{k}, r_{k}\right) \mid k \in I\right\} \backslash\{(a, b)\}$.

(1) Assume $|E| \geqslant 3$.

- If $\{a, a+1\} \subseteq\{x \mid \exists y(x, y) \in E\}$ and $\{(a, a+1),(a-1, a+1)\} \cap E \neq \varnothing$, then $p=a, r=b$. 
- Otherwise

$$
p=\max \{x \leqslant a+1 \mid \exists y(x, y) \in E\}
$$

and

$$
r= \begin{cases}b & \text { if }(a-1, b-1) \in E \text { or } \\ & a+1 \neq b-1 \in\{x \mid \exists y(x, y) \in E\} \\ b+1 & \text { otherwise. }\end{cases}
$$

(2) Assume $|E|=m \leqslant 2$. Then there exist $x, y \in[n-1]$ such that the following statements hold.

(i) $(a, b)=(p, r)$ if and only if

(A) $H(n)-m \leqslant n-b+1$ and $E \subseteq\{(a, b-1),(a-1, b-1),(a, x)$, $(b-1, y)\}$.

(ii) $(a, b)=(p, r-1)$ if and only if

(B) $H(n)-m \leqslant b-a+1$ and $E \subseteq\{(a, b+1),(a-1, b),(a, x),(b, y)\}$.

(iii) $(a, b)=(p-1, r-1)$ if and only if

(C) $H(n)-m \leqslant a$ and $E \subseteq\{(a+1, b+1),(a+1, b),(a+1, x),(b, y)\}$.

Proof. (I) This follows immediately from Lemma 13(i).

(II) Since, by Lemma 13(i), there may exist only one index $i \in[n]$ such that $\left(p_{i}, r_{i}\right)=$ $(r-1, x)$ for some $x \in[n]$, and only one index $j \in[n]$ such that $\left(p_{j}, r_{j}\right)=(p, y)$ for some $y \in[n] \backslash\{r-1, r\}$, we necessarily have

$$
\left\{\left(p_{\alpha}, r_{\alpha}\right),\left(p_{\gamma}, r_{\gamma}\right)\right\} \subseteq\{(p, r),(p, r-1),(p-1, r-1)\} .
$$

It follows that $p=\max \left\{p_{\alpha}, p_{\gamma}\right\}$ and $r-1=\min \left\{r_{\alpha}, r_{\gamma}\right\}$.

(III) We necessarily have $(a, b) \in\{(p, r),(p, r-1),(p-1, r-1)\}$. Table 3 shows the possible types of cards in terms of $a$ and $b$ in each case. Note that, by Lemma 13(i), the values of $x$ and $y$ are fixed and only depend on the positions of entries with values 1,2 , and 3 in $\pi$.

\begin{tabular}{cccccc}
\hline & $(p, r)$ & $(p, r-1)$ & $(p-1, r-1)$ & $(p, x)$ & $(r-1, y)$ \\
\hline Case 1 & $(a, b)$ & $(a, b-1)$ & $(a-1, b-1)$ & $(a, x)$ & $(b-1, y)$ \\
Case 2 & $(a, b+1)$ & $(a, b)$ & $(a-1, b)$ & $(a, x)$ & $(b, y)$ \\
Case 3 & $(a+1, b+1)$ & $(a+1, b)$ & $(a, b)$ & $(a+1, x)$ & $(b, y)$ \\
\hline
\end{tabular}

Table 3: The possible types of cards in the different cases of the proof of Lemma 16(III).

By our hypothesis we have $p<r-1$ and then $b-a \geqslant 2$ clearly holds in Cases 1 and 3. In Case 2 we have $(a, b)=(p, r-1)$ and since there are at least two cards of this type, we have $r-p-1 \geqslant 2$, so $b-a=r-p-1 \geqslant 2$ holds also in this case. Thus $b-a \geqslant 2$ always holds. 
(1) Assume $|E| \geqslant 3$. By reading off from Table 3, we can first note that the conditions $(a, b)=(p, r)$ and $b-1=a+1 \in\{x \mid \exists y(x, y) \in E\}$ hold simultaneously if and only if the conditions $\{a, a+1\} \subseteq\{x \mid \exists y(x, y) \in E\}$ and $\{(a, a+1),(a-1, a+1)\} \cap E \neq \varnothing$ also hold.

Now suppose $\{a, a+1\} \nsubseteq\{\{x \mid \exists y(x, y) \in E\}$ or $\{(a, a+1),(a-1, a+1)\} \cap E=\varnothing$. In Case 1 either $\{x \mid \exists y(x, y) \in E\}=\{a-1, a\}$ or $b-1>a+1$ and in both cases $p=a=\max \{x \leqslant a+1 \mid \exists y(x, y) \in E\}$ and $r=b$. In Cases 2 and 3, we can immediately conclude from Table 3 that $p=\max \{x \leqslant a+1 \mid \exists y(x, y) \in E\}$ and since neither $(a-1, b-1) \in E$ nor $b-1 \in\{x \mid \exists y(x, y) \in E\}$ with $b-1 \neq a+1$, we also have $r=b+1$.

(2) Assume $|E|=m \leqslant 2$. We prove first the necessity of conditions (A), (B), and (C).

If $(a, b)=(p, r)$, then the cards of type $(a, b)$ are among the cards $\pi-i$ with $r<i$ and, in the case with $\pi(r+1)=3$, also $\pi \downarrow 2=\pi-r$. Therefore, the inequality $H(n)-m \leqslant n-r+1=n-b+1$ holds. Furthermore, by reading off from Table 3, we see that $E \subseteq\{(a, b-1),(a-1, b-1),(a, x),(b-1, y)\}$ for some $x, y \in[n-1]$.

If $(a, b)=(p, r-1)$, then the cards of type $(a, b)$ are among the cards $\pi-i$ with $p<i<r$ and, in the case when $\pi(r-1)=3$, also $\pi \downarrow 2=\pi-r$. Therefore, the inequality $H(n)-m \leqslant r-p=b-a+1$ holds. Furthermore, by reading off from Table 3, we see that $E \subseteq\{(a, b+1),(a-1, b),(a, x),(b, y)\}$ for some $x, y \in[n-1]$.

If $(a, b)=(p-1, r-1)$, then the cards of type $(a, b)$ are among the cards $\pi-i$ with $i<p$; therefore the inequality $H(n)-m \leqslant p-1=a$ holds. Moreover, by reading off from Table 3, we see that $E \subseteq\{(a+1, b+1),(a+1, b),(a+1, x),(b, y)\}$ for some $x, y \in[n-1]$.

In order to prove the sufficiency of conditions (A), (B), and (C), it suffices to show that they are mutually exclusive. In the case when $m=2$, this is clear, because for any $x, y \in$ $[n-1]$, the intersection of any two of the sets $\{(a, b-1),(a-1, b-1),(a, x),(b-1, y)\}$, $\{(a, b+1),(a-1, b),(a, x),(b, y)\}$ and $\{(a+1, b+1),(a+1, b),(a+1, x),(b, y)\}$ has at most one element. Now assume that $m \leqslant 1$, and suppose, to the contrary, that some two of the inequalities $H(n)-m \leqslant n-b+1, H(n)-m \leqslant b-a+1$, and $H(n)-m \leqslant a$ hold simultaneously. Since $1 \leqslant a<b \leqslant n-1$ and $b-a \geqslant 2$, it follows that

$$
n+2 \leqslant 2(H(n)-m) \leqslant \max \{n-a+2, n-(b-a)+1, b+1\} \leqslant n+1,
$$

a contradiction.

If none of the $H(n)$ cards equals $\pi \downarrow 1$, then the next result assures that the $(n-2)$ patterns obtained by deleting the entry 1 from each card constitutes a partial deck of $\pi \downarrow 1$, and we can make use of this partial deck to reconstruct $\pi \downarrow 1$. Consequently, we can reconstruct $\pi$.

Lemma 17. Let $\pi \in S_{n}$. If $1<i_{1}<i_{2}<\cdots<i_{\ell} \leqslant n$, then $\left\langle\left(\pi \downarrow i_{j}\right) \downarrow 1 \mid j \in[\ell]\right\rangle$ is a partial deck of $\pi \downarrow 1$.

Proof. The multiset $\left\langle(\pi \downarrow 1) \downarrow\left(i_{j}-1\right) \mid j \in[\ell]\right\rangle$ is clearly a partial deck of $\pi \downarrow 1$, and $\left(\pi \downarrow i_{j}\right) \downarrow 1=(\pi \downarrow 1) \downarrow\left(i_{j}-1\right)$ holds by Lemma 5 .

Theorem 18. For every permutation $\pi$ of rank $n$, with $n \geqslant 5$, the value $\pi^{-1}(1)$ is uniquely determined by any partial $(n-1)$-deck $D$ of $\pi$ with $H(n)$ cards not all equal. Moreover, 
from the partial deck $D$ alone, it is also possible to decide whether any of the cards in $D$ is equal to $\pi \downarrow 1$, and, if positive, to identify such a card.

Proof. Let $D:=\left\langle\tau_{1}, \ldots, \tau_{H(n)}\right\rangle$ be a partial $n-1$-deck $D$ of $\pi$ with $H(n)$ cards and assume that $D$ has at least two distinct cards. Let $p:=\pi^{-1}(1)$ and $r:=\pi^{-1}(2)$. (Recall that we are given only the partial deck $D$, so $p$ and $r$ are unknown quantities.) By Lemma 13(ii) and (iii), we may determine whether $p<r$ or $p>r$ and whether $p=r-1$ by comparing the positions of 1 and 2 in the cards in $D$; in fact, it is enough to check only five cards (see Remark 14). Henceforth we may assume that $p<r$. Otherwise we may consider $D^{\mathrm{r}}:=\left\langle\tau_{1}^{\mathrm{r}}, \ldots, \tau_{H(n)}^{\mathrm{r}}\right\rangle$ instead of $D$ and reconstruct $\pi^{\mathrm{r}}$, which can then be reversed to obtain $\pi$. We now consider different cases and subcases.

Case 1: Assume $p=r-1$. By Lemma 15(i), $\pi \downarrow i=\pi \downarrow 1$ if and only if $\ell_{\pi \downarrow}=\ell_{\pi}-1$, so our aim is to determine $\pi^{-1}(1)$ and $\ell_{\pi}$, if possible. Then it will be straightforward to check whether or not $D$ contains a card $\tau_{k}$ with $\ell_{\tau_{k}}=\ell_{\pi}-1$; such a card equals $\pi \downarrow 1$.

Let $P:=\left\{\tau_{k}^{-1}(1) \mid k \in[H(n)]\right\}$. Then $\varnothing \neq P \subseteq\{p-1, p\}$. We consider two subcases according to the cardinality of $P$.

Case 1.1: Assume $|P|=2$. Then $P=\{p-1, p\}$, and we have $\ell_{\tau_{k}}=\ell_{\pi}$ for any $k$ such that $\tau_{k}^{-1}(1)=p-1$, by Lemma 15 (iii). Then it is easy to detect whether or not there is a card $\tau_{j}$ such that $\ell_{\tau_{j}}=\ell_{\pi}-1$, i.e., $\tau_{j}=\pi \downarrow 1$.

Case 1.2: Assume $|P|=1$, say $P=\{u\}$. Observe that, by Lemma 15, statements (iv) and $(\mathrm{v})$, either $u \geqslant H(n)$ or $n-(u-1) \geqslant H(n)$, and these cases are mutually exclusive.

Case 1.2.1: Assume $u \geqslant H(n)$. Then $p=u+1$ and $\pi \downarrow 1$ is not in $D$ by Lemma 15(iv).

Case 1.2.2: Assume $n-(u-1) \geqslant H(n)$. Then $p=u$ by Lemma 15(iv) and every card in $D$ must be of the form $\pi-t$ with $t \geqslant p$. Let $\pi[p, v]$ be the maximal monotone segment of length $\ell_{\pi}$ in $\pi$, let $L:=\left\langle\ell_{\tau_{j}}: j \in[H(n)]\right\rangle$, and let $k \in[H(n)]$ be such that $\ell_{\tau_{k}}=\min \operatorname{Supp}(L)$.

Observe that there is a number $a \geqslant 1$ such that $\varnothing \neq \operatorname{Supp}(L) \subseteq\left\{\ell_{\pi}-1, \ell_{\pi}, \ell_{\pi}+a\right\}$ with $\chi_{L}\left(\ell_{\pi}+a\right) \leqslant 2$ by Lemma 15(i) and (ii). If $\chi_{L}\left(\ell_{\pi}\right)=0$ holds, then $\chi_{L}\left(\ell_{\pi}-1\right)=$ $H(n)-\chi_{L}\left(\ell_{\pi}+a\right) \geqslant H(n)-2$; since $\chi_{L}\left(\ell_{\pi}-1\right) \leqslant \ell_{\pi}$, this implies $\ell_{\tau_{k}}=\ell_{\pi}-1 \geqslant H(n)-3$. If $\chi_{L}\left(\ell_{\pi}-1\right)=0$ holds, then $H(n)=\chi_{L}\left(\ell_{\pi}\right)+\chi_{L}\left(\ell_{\pi}+a\right) \leqslant n-v=n-\left(p-1+\ell_{\pi}\right)$ and so $\ell_{\tau_{k}}=\ell_{\pi} \leqslant n-H(n)-p+1$. Finally notice that we cannot have simultaneously $\ell_{\tau_{k}} \geqslant H(n)-3$ and $\ell_{\tau_{k}} \leqslant n-H(n)-p+1$ since $n-H(n)-p+1 \geqslant H(n)-3$ implies $n-p+1 \geqslant 2 H(n)-3 \geqslant n+1$.

Hence one of the following cases occurs.

Case 1.2.2.1: Assume $|\operatorname{Supp}(L)|=3$. Then $\ell_{\tau_{k}}=\ell_{\pi}-1$ and we have $\tau_{k}=\pi \downarrow 1 \in D$.

Case 1.2.2.2: Assume $|\operatorname{Supp}(L)| \leqslant 2$ and $\ell_{\tau_{k}} \geqslant H(n)-3$. Then $\ell_{\tau_{k}}=\ell_{\pi}-1$. Thus $\tau_{k}=\pi \downarrow 1 \in D$.

Case 1.2.2.3: Assume $|\operatorname{Supp}(L)| \leqslant 2$ and $\ell_{\tau_{k}} \leqslant n-H(n)-p+1$. Then $\ell_{\tau_{k}}=\ell_{\pi}$. Thus $\pi \downarrow 1 \notin D$.

Case 1.2.2.4: Assume $|\operatorname{Supp}(L)| \leqslant 2$ and neither of the inequalities $\ell_{\tau_{k}} \geqslant H(n)-3$ and $\ell_{\tau_{k}} \leqslant n-H(n)-p+1$ holds. By the above observations, we must then have $\chi_{L}\left(\ell_{\pi}-1\right)>0$ and $\chi_{L}\left(\ell_{\pi}\right)>0$; hence $L=\left\{\ell_{\pi}-1, \ell_{\pi}\right\}$ and $\ell_{\tau_{k}}=\ell_{\pi}-1$. Thus $\tau_{k}=\pi \downarrow 1 \in D$.

Case 2: Assume $p<r-1$. By Lemma 16(I), $\pi-t=\pi \downarrow 1$ if and only if $(\pi-t)^{-1}(1)=$ 
$r-1$, so our aim is to determine $\pi^{-1}(1)=p$ and $\pi^{-1}(2)=r$, if possible. Then it will be straightforward to check whether or not $D$ contains a card $\tau_{k}$ with $\tau_{k}^{-1}(1)=r-1$; such a card equals $\pi \downarrow 1$.

Let $T:=\left\langle\left(\tau_{k}^{-1}(1), \tau_{k}^{-1}(2)\right) \mid k \in H(n)\right\rangle$ be the multiset of the types of the cards in $D$. By Lemma 13, the only possible elements of $T$ are $(p, r),(p, r-1),(p-1, r-1),(r-1, x)$, and $(p, y)$, for some $x, y \in[n]$, and the only elements that may have multiplicity greater than 1 are $(p, r),(p, r-1)$, and $(p-1, r-1)$.

Case 2.1: Assume $T$ contains all five possible types. Since $p-1<p<r-1$, we can immediately determine both $p$ and $r-1$, and clearly $D$ contains a card $\tau_{k}$ with $\tau_{k}^{-1}(1)=r-1$ and so $\pi \downarrow 1=\tau_{k}$.

Case 2.2: Assume $T$ contains at most four different types. Since $H(n) \geqslant 5$, this means that some type has multiplicity at least 2 in $T$.

Case 2.2.1: Assume $(a, b)$ and $(c, d)$ are distinct types with multiplicity at least 2 in $T$. Then, by Lemma 16 (II), we have $p=\max \{a, c\}$ and $r-1=\min \{b, d\}$.

Case 2.2.2: Assume there is only one type $(a, b)$ of multiplicity at least 2 in $T$. Now the values of $p$ and $r-1$ are obtained by applying Lemma 16(III).

Theorem 19. For $n \geqslant 5$, every permutation of rank $n$ is reconstructible from $H(n)$ cards.

Proof. If all cards in $D$ are equal, say $\tau_{k}=\tau$ for all $k \in[H(n)]$, then by Lemma 7 (iii) $\tau$ contains a unique maximal monotone segment $\pi[u, v]$ of length at least $\lceil n / 2\rceil$, and by Lemma 7 (ii) we can immediately conclude that $\pi=\tau \uparrow_{u} \tau(u)$ if $\pi[u, v]$ is ascending and $\pi=\tau \uparrow_{v+1} \tau(v)$ if $\pi[u, v]$ is descending.

From now on we may assume that $D$ has at least two distinct cards. We prove the claim by induction on $n$. For $n=5$, we have $H(n)=5$, and the claim holds by the results of Smith [6, Theorem 2.3] and Raykova [5, Lemma 3.3]. Assume now that every permutation of rank $m(m \geqslant 5)$ is reconstructible from $H(m)$ cards. Let $\pi \in S_{m+1}$, and let $D=\left\langle\tau_{1}, \ldots, \tau_{H(m+1)}\right\rangle$ be a partial $m$-deck of $\pi$ with $H(m+1)$ cards. By Theorem 18 we can find $\pi^{-1}(1)$ and we can determine if $\pi \downarrow 1 \notin D$.

Case 1: Assume that $\pi \downarrow 1 \notin D$. In this case, $D^{\prime}:=\left\langle\tau_{k} \downarrow 1 \mid k \in[H(m+1)]\right\rangle$ is a partial deck of $\pi \downarrow 1$ by Lemma 17. Since $\left|D^{\prime}\right|=H(m+1) \geqslant H(m)$, our inductive hypothesis asserts that we can reconstruct $\pi \downarrow 1$ from $D^{\prime}$, and then we recover $\pi$ as $\pi=(\pi \downarrow 1) \uparrow_{\pi^{-1}(1)} 1$ by Lemma 4 .

Case 2: Assume that $\pi \downarrow 1 \in D$. In this case we may identify $\pi \downarrow 1$ among the cards in $D$, by Theorem 18. Then we recover $\pi$ as $\pi=(\pi \downarrow 1) \uparrow_{\pi^{-1}(1)} 1$ by Lemma 4 .

The proofs of Theorems 18 and 19, along with Lemmas 15 and 16, give readily rise to a reconstruction algorithm for an unknown permutation $\pi$ if any of its partial decks of size $H(n)$ is given. We may also make use of the shortcuts provided by Propositions 8 and 12. The following examples illustrate the reconstruction method.

\section{Example 20.}

1. Let $\pi \in S_{10}$ and take $D$ to be the following partial deck of $\pi$ :

$$
\left\langle(978612354)^{3},(987123654)^{2},(978123654)^{1},(897123654)^{1}\right\rangle .
$$


Hence $D=\operatorname{deck}_{I}(\pi)$ for some $I \subseteq[10]$ with $|I|=7=H(10)$. The sequence $\sigma=123$ is a maximal ascending segment in every card in $D$. We apply Proposition 12 . From part (ii) we obtain the following quantities: $G=\{(1,5),(1,4)\}, A=\{1\}$, $B=\{4,5\}, a^{*}=1, b^{*}=5, t=a^{*}=1, u=b^{*}=5, v=5+3-1=7$. Consequently, $\pi[5,7]=123$ is a maximal ascending segment in $\pi$.

Part (iii) yields the partial deck $D^{\prime}=\left\langle(645321)^{4},(654321)^{2},(564321)^{1}\right\rangle$ of $\pi \downarrow \pi[5,7]$. We apply Lemmas 6 and 7 (ii) to the card $\tau=645321$ of multiplicity 4 , and we obtain $\pi \downarrow \pi[5,7]=\tau \uparrow_{7} \tau(6)=645321 \uparrow_{7} 1=7564321$. Hence $\pi=(\pi \downarrow \pi[5,7]) \uparrow_{5} \pi[5,7]=7564321 \uparrow_{5} 123=10897123654$.

2. Let $\pi \in S_{11}$ and let $D$ be the partial deck of $\pi$ comprising

$$
\begin{array}{ll}
\tau_{1}=10246831759, & \tau_{2}=10136842759, \\
\tau_{3}=10135694279, & \tau_{4}=10124683759, \\
\tau_{5}=10135784269, & \tau_{6}=10134682759, \\
\tau_{7}=13579428610, & \tau_{8}=10135794286 .
\end{array}
$$

First we compare the positions of 1 and 2 in the cards. With the help of Lemma 13(ii) and (iii) we conclude that $p:=\pi^{-1}(1)<\pi^{-1}(2)=: r$ and $p<r-1$. The multiset of pairs $\left(\tau^{-1}(1), \tau^{-1}(2)\right)$ with $\tau \in D$ is $\left\langle(1,7)^{1},(2,3)^{1},(2,7)^{2},(2,8)^{3},(7,2)^{1}\right\rangle$. This multiset contains two distinct elements of multiplicity greater than 1 , namely $(2,3)$ and $(2,7)$, so we can apply Lemma 16 (II) and we obtain $\left(\pi^{-1}(1), \pi^{-1}(2)\right)=$ $(\max \{2,2\}, \min \{3,7\}+1)=(2,8)$. Since $\tau_{1}^{-1}(1)=7=\pi^{-1}(2)-1$ we have $\tau_{1}=\pi \downarrow 1$ by Lemma $16(\mathrm{I})$, and then $\pi=\tau_{1} \uparrow_{2} 1=1113579428610$.

3. Let $\pi \in S_{8}$, and let $D=\left\langle(5741326)^{3},(6751432)^{1},(5743216)^{1},(5714326)^{1}\right\rangle$. Applying Lemma 13, we conclude that $p:=\pi^{-1}(1)<\pi^{-1}(2)=: r$ and $p<r-1$. The multiset of pairs $\left(\tau^{-1}(1), \tau^{-1}(2)\right)$ with $\tau \in D$ is $\left\langle(4,6)^{3},(4,7)^{1},(6,5)^{1},(3,6)^{1}\right\rangle$. This multiset has a unique element of multiplicity greater than 1 , namely $(4,6)$, and three elements of multiplicity 1, so we apply Lemma 16(III)(1) and we obtain $\pi^{-1}(1)=p=4$ and $\pi^{-1}(2)=r=7$. Since $\tau=5743216 \in D$ satisfies $\tau^{-1}(1)=$ $6=r-1$, we have $\pi=\tau \uparrow_{4} 1$ by Lemma 16(I). Hence $\pi=68514327$.

4. Let $D$ be the following partial deck of $\pi \in S_{9}$ :

$$
\left\langle(17283456)^{1},(71283456)^{1},(81723456)^{1},(71628345)^{4}\right\rangle .
$$

By applying Lemma 13 we conclude that $p:=\pi^{-1}(1)<\pi^{-1}(2)=: r$ and $p<r-1$. The multiset of pairs $\left(\tau^{-1}(1), \tau^{-1}(2)\right)$ with $\tau \in D$ is $\left\langle(1,3)^{1},(2,3)^{1},(2,4)^{5}\right\rangle$. This multiset has a unique element of multiplicity greater than 1 , namely $(a, b):=(2,4)$, and $m:=2$ elements of multiplicity 1 , so with the help of Lemma 16(III)(2) we conclude that $(p, r)=(2,4)$ since $n-b=9-4=5 \geqslant 7-2=H(n)-m$ and there is no $(x, y) \in D$ with $x=b=4$. No card in $D$ has 1 at position $r-1=3$ and so $\pi \downarrow 1 \notin D$ by Lemma 16(I). 
Now take

$$
D^{\prime}:=\langle(\pi \downarrow i) \downarrow 1 \mid i \in I\rangle=\left\langle(6172345)^{2},(7612345)^{1},(6517234)^{4}\right\rangle ;
$$

$D^{\prime}$ is a partial deck of $\pi \downarrow 1 \in S_{8}$ of cardinality $H(8)+1$. Theorem 19 guarantees that $\theta=\pi \downarrow 1$ is reconstructible from the multiset

$$
D_{\theta}=\left\langle(6172345)^{1},(7612345)^{1},(6517234)^{4}\right\rangle \subseteq D^{\prime} .
$$

We apply Proposition 8 to the card $\tau=6517234$ that has multiplicity 4 and contains a unique maximal monotone segment of length 3 , namely $\tau[5,7]=234=$ : $\sigma$. Since $\sigma$ is ascending, we get $\theta=\tau \uparrow_{5} \tau(5)=\tau \uparrow_{5} 2=76182345$. Finally we obtain $\pi=\theta \uparrow_{p} 1=\theta \uparrow_{2} 1=817293456$.

\section{Concluding remarks and open problems}

We have taken the first steps of answering Ginsburg's Problem 2 by showing that $H_{1}(n)=$ $\lceil n / 2\rceil+2$ for all $n \geqslant 5$. It remains an open problem to determine values of $H_{k}(n)$ when the parameter $k$ is greater than 1 .

Another curious problem, which may be a necessary step for approaching Problem 2, is to generalize Ginsburg's Lemma 6 and determine necessary and sufficient conditions for the equality of two $(n-k)$-cards of an $n$-permutation when $k \geqslant 2$. In this case, the situation looks much more complicated. It is possible that two cards coincide even if entries are not removed from the same monotone segments, as illustrated by the following example: there are no nontrivial monotone segments in the permutation $\pi=52413$, yet we have $\pi \downarrow\{1,3\}=\pi \downarrow\{1,4\}=\pi \downarrow\{2,4\}=\pi \downarrow\{2,5\}=312$.

\section{References}

[1] M. Bóna. Combinatorics of Permutations. Discrete Math. Appl. (Boca Raton). Chapman \& Hall/CRC, Boca Raton, 2004.

[2] J. Ginsburg. Determining a permutation from its set of reductions. Ars Combin., 82:55-67, 2007.

[3] P. J. Kelly. On Isometric Transformations. Ph.D. thesis, University of Wisconsin, 1942.

[4] S. Kitaev. Patterns in Permutations and Words. Monogr. Theoret. Comput. Sci. EATCS Ser. Springer, Heidelberg, 2011.

[5] M. Raykova. Permutation reconstruction from minors. Electron. J. Combin., 13:\#R66, 2006.

[6] R. Smith. Permutation reconstruction. Electron. J. Combin., 13:\#N11, 2006.

[7] S. M. Ulam. A Collection of Mathematical Problems. Interscience Tracts in Pure and Applied Mathematics, no. 8. Interscience Publishers, New York, 1960. 